\title{
Relationship between core body temperature and heart rate variability in an ultrashort sleep-wake schedule
}

https://doi.org/10.1515/cdbme-2019-0012

\begin{abstract}
The circadian rhythm that drives the human sleepwake cycle manifests itself in the variation of the core body temperature (CBT). Measuring CBT continuously, however, is intricate. Heart rate and its variability also varies notably over the course of the day, but is more accessible to measure. We present first results from a study that investigates the biorhythmic relationship between the circadian rhythm of CBT and measures of heart rate variability (HRV). Twelve subjects participated in a 50-hour experiment that was segmented into one hundred 30-minute blocks. In each block, subjects attempted to sleep for ten minutes. CBT and the ECG were continuously recorded. To date, intervals between successive heart beats (RR intervals) were determined for three subjects. From RR intervals of each attempted-sleep episode, power spectral densities (PSD) were estimated with the Lomb-Scargle periodogram and averaged into $0.02-\mathrm{Hz}$ wide bands. The periodogram of each RR-frequency-band time series was estimated and compared against the periodogram of CBT, which, as expected, shows a dominant maximum at a period length of 24 hours. Mutual information, an entropy-based measure of interdependence, was used for the comparison. The highest coherence towards CBT was found for the RR-PSD between 0.24 and $0.34 \mathrm{~Hz}$. This RR-PSD range usually includes the respiratory rate, which may indicate that heart rate is more closely linked to respiration at certain times of the day than at others.
\end{abstract}

Keywords: Heart rate variability, core body temperature, circadian rhythm, Lomb-Scargle periodogram, mutual information

\section{Introduction}

Chronobiology and sleep research has established that the human sleep-wake cycle is driven by endogenous physiological clocks which maintain their 24-hour oscillation even when isolated from external influences and cues. This circadian rhythm

*Corresponding author: Christian Heinze, University of Applied Sciences Schmalkalden, Blechhammer 4-9, Schmalkalden, Germany, e-mail: c.heinze@hs-sm.de

Martin Golz, University of Applied Sciences Schmalkalden, Schmalkalden, Germany manifests itself e. g. in the variation of the core body temperature [1] or the melatonin blood levels [2]. In healthy human, sleep-wake times are closely linked to these processes. Reversely, individual sleep-wake times can be estimated when properties of an individual's circadian rhythm, particularly the circadian phase, are known. Such sleep-wake estimations could help to model the periods of wakefulness and fatigue, which is important (and particularly difficult) for occupational groups where the work activity strongly influences the circadian rhythm, such as shift workers or pilots.

Measuring variables like core body temperature (CBT) or blood melatonin, however, is not straightforward, and difficult outside a laboratory. A more accessible variable would be heart rate and its variability, which also varies notably over the course of the day. This doesn't refer to the obvious fact that the heart beats faster and with greater ejection volume during the day due to higher exertion, but to the phenomenon known as heart rate variability, according to which the time difference between successive heart beats varies even at rest [3].

We present first results from a more comprehensive study which aims to reveal biorhythmic relationships between different physiological variables-particularly, in this case, between the circadian rhythm of CBT on one hand, and measures of heart rate variability (HRV) on the other. We investigated which measures of HRV can suitably estimate the period length of the circadian rhythm, comparable to the CBT.

We subjected our volunteers to a 50-hour long experiment that was segmented into one hundred 30-minute blocks. In each, a subject attempted to fall asleep for ten minutes. After wake-up, sleep was not allowed in the remaining 20-minute period. In chronobiology reserach, such a setup is called $u l$ trashort sleep-wake schedule [4-6]. Originally, it was used to investigate the sleep latency as a function of the time of day, where the researchers segmented the experiment into 20minute blocks ( $7 \mathrm{~min}$ of attempted sleep, followed by $13 \mathrm{~min}$ of wakefulness) over a duration of 24 hours. Since our goal is to estimate a circadian period length around 24 hours, we extended the experiment duration to 50 hours, while stretching the block length to $30 \mathrm{~min}$. This provided us with 100 samples (per subject) of 10-min episodes where the subjects were resting and where, therefore, the heart beat was unaffected by exertion or other distorting influences. 

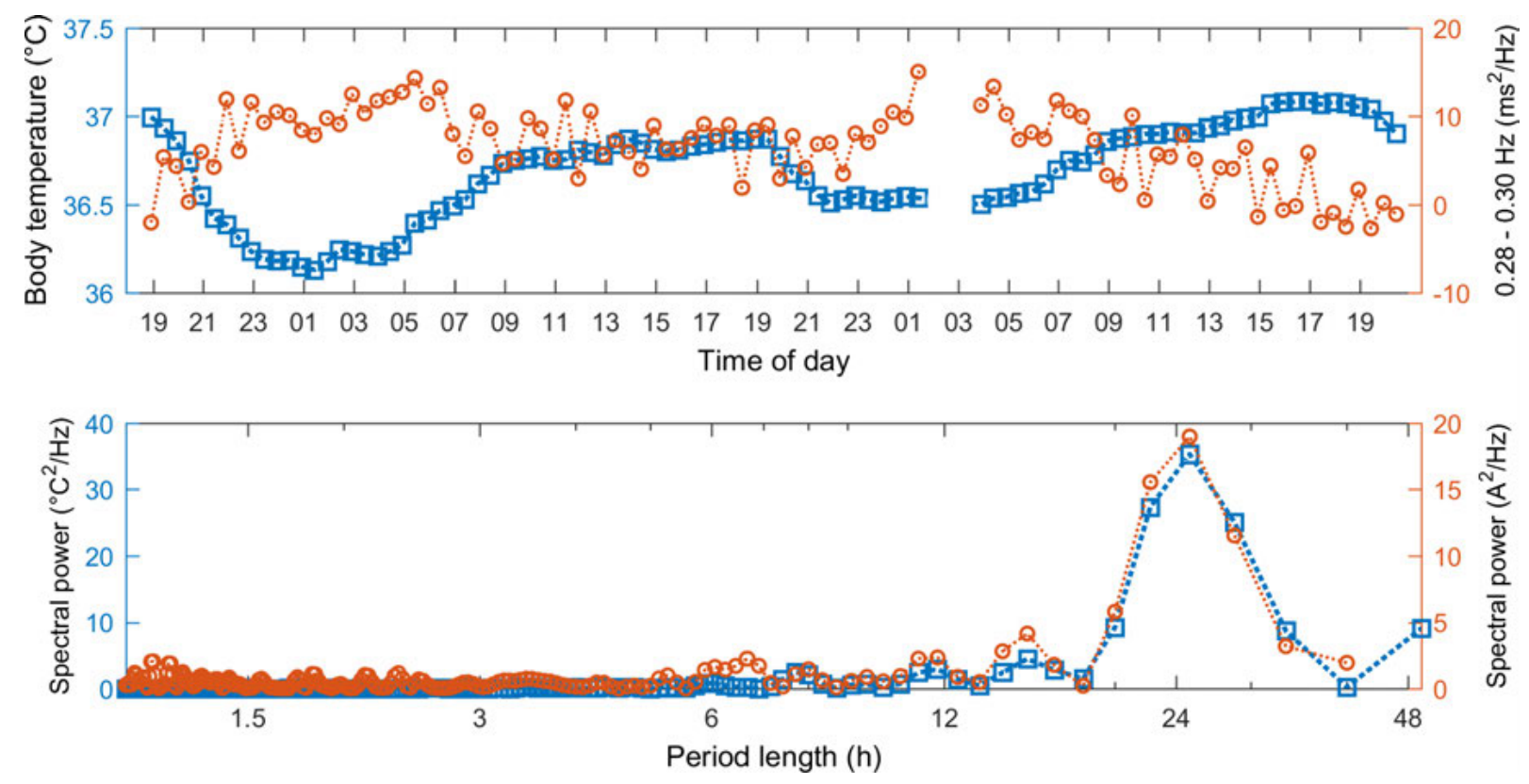

Fig. 1: Example of the relationship between CBT and RR-PSD. Upper part: Course of the CBT (blue squares) and the RR-PSD between 0.28 and $0.3 \mathrm{~Hz}$ (red circles) during 50 hours for subject 3. Each data point corresponds to a 10-minute episode of attempted sleep, in which the CBT values were averaged and the spectral power of RR intervals between 0.28 and $0.3 \mathrm{~Hz}$ was estimated, respectively. (The gap in both series represents a data loss a three hours.) Lower part: The Lomb-Scargle power spectral density estimate of the CBT and the RR $0.28-0.3-\mathrm{Hz}$ PSD band. Both spectra are normalized by the variance of their signals. The absize was converted from frequency $(\mathrm{Hz})$ to period length $(\mathrm{h})$. Clearly, the RR $0.28-0.3-\mathrm{Hz}$ PSD band reflects the 24-h circadian rhythm, comparable to the CBT. (Note that both spectra are scaled individually; compared to CBT, the RR-PSD has only half the spectral power.)

\section{Material}

Twelve subjects ( 5 female, 7 male, 18 to 30 years old) took part in the 50-hour experiment described above, during which CBT, ECG, EEG, and EOG were continuously recorded. The CBT was recorded with an ear temperature sensor connected to a portable recording device (Somnowatch plus, sampling rate: $1 \mathrm{~s}^{-1}$, resolution: $0.01 \mathrm{~K}$ ). This measurement method extrapolates the CBT at the tympanic membrane using two temperature signals that yield a linear estimate of the temperature along the ear canal. A one-channel ECG was recorded with a polygraphy device (Somnoscreen EEG-10-20, sampling rate: $512 \mathrm{~s}^{-1}$ ). The EEG/EOG were recorded with the same device, they are intended for sleep-stage classification in future analyses and not considered here.

The intervals between successive heart beats (RR intervals) were determined from the ECG recordings of, to date, three subjects. For R-peak detection, we used an algorithm [7] that adopts the widely used procedure of Pan \& Tompkins [8] but employs some improvements which increases its robustness towards noise and signal drifts. The automatic detections were then reviewed; false-positive and false-negative peaks were removed and added, respectively, so that RR intervals were determined only between clear, normal $\mathrm{R}$ peaks.

\section{Methods}

From the RR-interval time series of each 10-min attemptedsleep episode, the power spectral densities (PSD) were estimated. The PSD of RR intervals (will be referred to as RRPSD from here on) is known to be strongest between 0 and $0.4 \mathrm{~Hz}$ and contains a broad spectrum [5]. Spectral estimators like the discrete Fourier transform or auto-regressive modeling assume that data is sampled at a constant rate. RR-interval series, however, are irregularly spaced by nature. We therefore applied the Lomb-Scargle periodogram $[6,7]$ for spectral estimation, a technique that was created specifically for unevenly sampled data. The spectral power $P$ of data $X$ at frequency $\omega$ is defined as:

$$
\begin{array}{r}
P_{X}(\omega)=\frac{1}{2}\left\{\frac{\left[\sum_{j} x_{j} \cos \omega\left(t_{j}-\tau\right)\right]^{2}}{\sum_{j} x_{j} \cos ^{2} \omega\left(t_{j}-\tau\right)}+\right. \\
\left.\frac{\left[\sum_{j} x_{j} \sin \omega\left(t_{j}-\tau\right)\right]^{2}}{\sum_{j} x_{j} \sin ^{2} \omega\left(t_{j}-\tau\right)}\right\},
\end{array}
$$


where $\tau$ is defined by

$$
\tan (2 \omega \tau)=\frac{\left(\sum_{j} \sin 2 \omega t_{j}\right)}{\left(\sum_{j} \cos 2 \omega t_{j}\right)}
$$

This definition is equivalent to least-squares fitting sine waves to the data, i. e. a maximum in the periodogram occurs at a frequency $\omega$ which minimizes the sum of squares of the residuals. This will yield the same spectral estimation for evenly sampled data as the classical periodogram. Unlike the classical periodogram, however, the Lomb-Scargle periodogram will not introduce spurious spectral peaks for unevenly sampled data that could erroneously indicate a periodic component.

After spectral estimation, the periodogram of each RRinterval time series contained several hundred data points. To reduce them to digestable features, each $\mathrm{RR}$ periodogram was averaged into (a) the very-low $(<0.04 \mathrm{~Hz})$, low $(0.04-0.15$ $\mathrm{Hz})$ and high $(0.15-0.4 \mathrm{~Hz})$ frequency bands that are conventionally used for RR analysis [11], and (b) into twenty 0.02$\mathrm{Hz}$ wide frequency bands, ranging from 0 to $0.4 \mathrm{~Hz}$, which allowed for a finer-grained analysis.

From each 50-hour CBT recording, the perdiogram was estimated (also using the Lomb-Scargle method, since that data contained gaps). As expected, it exhibits a dominant maximum at a period length of 24 hours, which clearly shows that CBT changes are driven by the circadian biological rhythm (see fig. 1, where the CBT recording of one subject is displayed in the time and the frequency domain). Then, from each RR-PSD feature (described in the previous paragraph) of each 10-min attempted-sleep episode, a 50-hour time series was created, from which, in turn, the periodogram was estimated. For each subject, the CBT periodogram was compared to the periodogram of each RR-PSD feature, in order to determine which RR feature exhibits a circadian rhythm similar to the CBT.

As a measure of interdependence between two variables, we used mutual information, which is based on information theory and entropy [12]. The entropy of a random variable is a measure of its uncertainty, i.e. the amount of information required on the average to describe that random variable. Mutual information is a measure of the amount of information that one random variable contains about another. It is the reduction in the uncertainty of one random variable due to the knowledge of the other one. In contrast to the linear correlation coefficient, mutual information is sensitive to dependences which are not reflected in the covariance. For two variables $X$ and $Y$ with a joint probability mass function $\mu(x, y)$ and marginal probability mass functions $\mu(x)$ and $\mu(y)$, the mutual information $I(X, Y)$ is the relative entropy (a measure of the distance between two distributions) between the joint distribution and the product distribution $\mu(x) \mu(y)$ :

$$
I(X, Y)=\sum_{x \in X} \sum_{y \in Y} \mu(x, y) \log \frac{\mu(x, y)}{\mu(x) \mu(y)}
$$

\section{Results}

After each 50-hour RR-PSD feature series had been assigned its mutual information towards the CBT periodogram, the RR features were sorted by their interdependence in descending order. For each subject, table 1 shows the top-five RR features whose periodograms resemble that of the CBT best. Across subjects, the most similar RR features were amongst the high frequency range-more precisely, between 0.24 and $0.34 \mathrm{~Hz}$. The relationship of these particular RR-PSD bands towards the CBT was anticyclical: when CBT was low, those RR-PSD values were high, and vice versa, as the upper part of fig. 1 exemplifies.

RR-PSD features within the high-frequency band weren't the only ones with a dominant 24-h frequency component. For subject 2, RR-PSD features from the very-low frequency band also exhibited a strong coherence towards CBT: the very-low frequency band itself, and the RR 0.02-0.04-Hz band (which is part of the very-low frequency band). Their periodograms also exhibited a dominant near-24-h maximum; because these maxima were located at period lengths slightly smaller than 24 hours, the agreement with the CBT periodogram was lower than for other RR-PSD features. The relationship of these very-low RR-PSD features towards the CBT was anticyclical as well.

To determine the RR-PSD features with the strongest CBT coherence across all subjects, the average rank for each RR feature was determined. For example, the RR 0.28-0.30$\mathrm{Hz}$ band has been ranked $1^{\text {st }}$ twice (subjects 1 and 3 ) and $5^{\text {th }}$ once (subject 2); this yields an average rank of $2.33 \pm$ a halfrange of 2 . As table 2 shows, the RR $0.28-0.30-\mathrm{Hz}$ band has been the highest ranking feature across subjects, followed by four other RR-PSD bands between 0.24 and $0.34 \mathrm{~Hz}$.

\section{Discussion}

The frequency interval between 0.24 and $0.34 \mathrm{~Hz}$, where, in our analysis, the RR-PSD showed the strongest coherence towards the CBT periodogram and, inferentially, towards the circadian biological rhythm, usually includes the respiratory rate as well. For example, a heart rate of $0.3 \mathrm{~s}^{-1}$ corresponds to 18 $\mathrm{min}^{-1}$, and 18 respirations/min in turn is the normal respiratory rate for an adult at rest. The respiratory rate usually shows up 
Tab. 1: For each subject, the RR-PSD features (left columns) with the strongest interdependence to the PSD of the subject's CBT are shown. Mutual information ( \pm standard error, right columns) is used as a measure of interdependence. The feature low/high frequency (subject 1) refers to the normalized ratio of power between the low and the high RR frequency band.

\begin{tabular}{|c|c|c|c|c|c|}
\hline \multicolumn{2}{|c|}{ Subject 1} & \multicolumn{2}{|c|}{ Subject 2} & \multicolumn{2}{|c|}{ Subject 3} \\
\hline $0.28-0.30 \mathrm{~Hz}$ & $0.17 \pm 0.06$ & $0.24-0.26 \mathrm{~Hz}$ & $0.13 \pm 0.05$ & $0.28-0.30 \mathrm{~Hz}$ & $0.19 \pm 0.06$ \\
\hline $0.32-0.34 \mathrm{~Hz}$ & $0.14 \pm 0.05$ & $0.26-0.28 \mathrm{~Hz}$ & $0.13 \pm 0.05$ & $0.30-0.32 \mathrm{~Hz}$ & $0.18 \pm 0.06$ \\
\hline $0.30-0.32 \mathrm{~Hz}$ & $0.14 \pm 0.05$ & Very-low frequency & $0.12 \pm 0.05$ & $0.26-0.28 \mathrm{~Hz}$ & $0.18 \pm 0.05$ \\
\hline Low/high frequency & $0.12 \pm 0.05$ & $0.02-0.04 \mathrm{~Hz}$ & $0.12 \pm 0.05$ & $0.32-0.34 \mathrm{~Hz}$ & $0.17 \pm 0.06$ \\
\hline $0.24-0.26 \mathrm{~Hz}$ & $0.12 \pm 0.05$ & $0.28-0.30 \mathrm{~Hz}$ & $0.12 \pm 0.05$ & $0.34-0.36 \mathrm{~Hz}$ & $0.16 \pm 0.05$ \\
\hline
\end{tabular}

Tab. 2: RR frequency features with the strongest interdependence with the PSD of the CBT for all three subjects, determined by their average rank \pm half their rank range.

\begin{tabular}{rl}
\hline RR feature & Average rank \\
\hline $0.28-0.30 \mathrm{~Hz}$ & $2.33 \pm 2$ \\
$0.32-0.34 \mathrm{~Hz}$ & $4.66 \pm 3$ \\
$0.26-0.28 \mathrm{~Hz}$ & $5.66 \pm 5$ \\
$0.24-0.26 \mathrm{~Hz}$ & $6 \quad \pm 5.5$ \\
$0.30-0.32 \mathrm{~Hz}$ & $9.66 \pm 11$ \\
\hline
\end{tabular}

in the RR-PSD as a local maximum, since respiration modulates the instantanious heart rate. This linkage between respiratory and heart rate is known as respiratory sinus arrhythmia [13]. The fact that RR interval changes between 0.24 and 0.34 $\mathrm{Hz}$ exhibit a 24-hour rhythm analogous to CBT may indicate that heart rate is more closely linked to respiration at certain times of the day than at others. Therefore, in order to estimate properties of the circadian rhythm, it may be benefical to include respiration data into the analysis.

It remains to be seen whether the found relationship between high-frequency HRV and CBT will persist when all subjects are included. In further analysis, the sleep latency, i. e. the time need to reach sleep stage II, will be estimated for each 10min attempted-sleep episode. This will allow insights into the connection between HRV dynamics and the circadian rhythm of sleepiness. On the outlook, it would be worthwhile to investigate if the found relationship can also be established in a less controlled environment, when the data is not recorded throughout the entire day and under controlled conditions, but for example only at night, during normal sleep.

\section{References}

[1] Zulley J., Wever R., \& Aschoff J. (1981). The dependence of onset and duration of sleep on the circadian rhythm of rectal temperature. Pflügers Archiv, European Journal of Physiology, 391(4), 314-18.

[2] Arendt J. (2000). Melatonin, Circadian Rhythms, and Sleep. New England Journal of Medicine, 343(15), 1114-16.
[3] Kräuchi K. \& Wirz-Justice A. (1994). Circadian rhythm of heat production, heart rate, and skin and core temperature under unmasking conditions in men. American Journal of Physiology-Regulatory, Integrative and Comparative Physiology, 267(3), R819-29.

[4] Lavie P. \& Scherson A. (1981). Ultrashort sleep-waking schedule. I. Evidence of ultradian rhythmicity in sleepability. Electroencephalography and Clinical Neurophysiology, 52(2), 163-74.

[5] Lavie P. \& Zomer J. (1984). Ultrashort sleep-waking schedule. II. Relationship between ultradian rhythms in sleepability and the REM-non-REM cycles and effects of the circadian phase. Electroencephalography and clinical neurophysiology, 57(1), 35-42.

[6] Lavie P. (1986). Ultrashort sleep-waking schedule. III. Gates and forbidden zones for sleep. Electroencephalography and clinical neurophysiology, 63(5), 414-25.

[7] Manikandan M. S. \& Soman K. P. (2012). A novel method for detecting R-peaks in eletrocardiogram signals. Biomedical Signal Processing and Control, 7(2), 118-28.

[8] Pan J. \& Tompkins W. J. (1985). A real-time QRS detection algorithm. IEEE Transactions on Biomedical Engineering, 32(3), 230-36.

[9] Lomb N. R. (1976). Least-squares frequency analysis of unequally spaced data. Astrophysics and space science, 39(2), 447-62.

[10] Scargle J. D. (1982). Studies in astronomical time series analysis. II-Statistical aspects of spectral analysis of unevenly spaced data. The Astrophysical Journal, 263, 835-53.

[11] Camm A. J., Malik M., Bigger J. T., Breithardt G., Cerutti S., Cohen R. J., Coumel P., Fallen E. L., Kennedy H. L., Kleiger, R. \& Lombardi F. (1996). Heart rate variability: standards of measurement, physiological interpretation and clinical use. Task Force of the European Society of Cardiology and the North American Society of Pacing and Electrophysiology.

[12] Thomas J. A. \& Cover T. M. (1991). Elements of information theory. Wiley, New York. Section 2.3, pp. 19.

[13] Ludwig C. (1847). Beiträge zur Kenntniss des Einflusses der Respirationsbewegungen auf den Blutlauf im Aortensysteme. Archiv für Anatomie, Physiologie und wissenschaftliche Medicin, 13, 242-302. 